\begin{tabular}{|c|c|}
\hline Title & $\begin{array}{l}\text { A New Mesh Smoothing Method to Improve the Condition Number of Submatrices of Coefficient Matrix in Edge } \\
\text { Finite Element Method }\end{array}$ \\
\hline Author(s) & Noguchi, So; T akada, A tsushi; Nobuyama, Fumiaki; Miwa, Masahiko; Igarashi, Hajime \\
\hline Citation & $\begin{array}{l}\text { IEEE Transactions On Magnetics, 49(5), 1705-1708 } \\
\text { https://doi.org/10.1109/T MA G.2013.2239978 }\end{array}$ \\
\hline Issue Date & $2013-05$ \\
\hline Doc URL & http:/hdl.handle.net/2115/53268 \\
\hline Rights & $\begin{array}{l}\text { (c) } 2013 \text { IEEE. Personal use of this material is permitted. Permission from IEEE must be obtained for all other uses, in } \\
\text { any current or future media, including reprinting/republishing this material for advertising or promotional purposes, } \\
\text { creating new collective works, for resale or redistribution to servers or lists, or reuse of any copyrighted component of } \\
\text { this work in other works. }\end{array}$ \\
\hline Tyре & article (author version) \\
\hline File Information & Noguchi_revised.pdf \\
\hline
\end{tabular}

Instructions for use 


\title{
A New Mesh Smoothing Method to Improve the Condition Number of Submatrices of Coefficient Matrix in Edge Finite Element Method
}

\author{
So Noguchi ${ }^{1}$, Atsushi Takada ${ }^{1}$, Fumiaki Nobuyama ${ }^{1}$, Masahiko Miwa², and Hajime Igarashi $^{1}$ \\ ${ }^{1}$ Graduate School of Information Science and Technology, Hokkaido University, Sapporo 060-0814, Japan \\ ${ }^{2}$ Electromagentic Engineering Department, Engineering Technology Division, JSOL Corporation, Osaka 550-0001, Japan
}

\begin{abstract}
A common mesh smoothing method strives to improve the shape quality of all elements. Generally a mesh consisting of only wellshaped elements is desired in finite element analysis. Although a perfect-shaped element yields short computation time, even a wellshaped element, whose shape is close to a regular polygon, sometimes prolongs the computation time of solving the system of equations derived with the edge-based finite element method. In this paper, we propose a new smoothing scheme of improving a convergence property of the system of equations by applying a common mesh smoothing method to some elements, which cause long computation time of the iterative solver. The proposed smoothing scheme utilizes the condition number of submatrices, into which coefficient matrix derived with the edge-based finite element method is subdivided, in order to choose ill-conditioned elements to be smoothed. As a result, the computation time is shortened applying a smoothing process only to the chosen ill-conditioned elements.
\end{abstract}

Index Terms - Coefficient matrix, finite element method, mesh generation, mesh smoothing.

\section{INTRODUCTION}

$\mathrm{T}$ HE QUALITY OF MESH is one of the most important factors when a finite element analysis (FEA) is performed. It strongly affects the computation time of FEA, hence a highquality mesh is desired usually. To generate such a highquality mesh, many mesh generation, mesh refinement, and adaptive meshing methods have been proposed [1]-[3]. A common mesh generation system contains a mesh smoothing process. A common mesh smoothing method represented by the Laplacian smoothing method [4] strives to deform all elements in an entire region close to a regular polygon. Consequently, the common mesh smoothing methods averagely improve the shape of all the elements in the entire mesh. That is, the shape of bad-shaped elements is improved, whereas the shape of well-shaped elements may be deteriorated. On the other hand, some mesh generation, such as the dynamic bubble system [5], strives to directly generate a high-quality mesh containing many well-shaped elements. Here, the well-shape element means a regular tetrahedron. However, when the edge-based finite element method (FEM) is employed for 3-D electromagnetic simulation, the orthogonality of the edges of elements, i.e. three edges of six are orthogonal each other in the case of a tetrahedral mesh, is an important factor in general [6]. The papers [6]-[8] reported that a regular tetrahedron was the most suitable element for the edge-based FEM, but a tetrahedral element, whose height was slightly shorter than that of regular tetrahedron, illaffected the convergence property of an iterative solver, such as the ICCG method. However, it is not clearly revealed what shape of element ill-affects the convergence property of the ICCG method. Therefore, a common geometrical evaluation method of element shape (e.g. ratio between the radius of the inscribed and that of the circumscribed sphere) is unuseful and ineffective [9].

At the point of view different from geometrical shape, a

Manuscript received October 31, 2012. Corresponding author: S. Noguchi (e-mail: noguchi@ssi.ist.hokudai.ac.jp)

Digital Object Identifier inserted by IEEE method of evaluating the property of coefficient matrix derived with the edge-based FEM was proposed [10]. The condition number of coefficient matrix represents the quality of the coefficient matrix to solve it with the iterative solver. Moreover, the quality of the coefficient matrix is able to be evaluated from the condition number of submatrices into which the coefficient matrix is subdivided [11].

In this paper, the condition number of submatrices is adopted for evaluating the quality of elements which correspond to the submatrices. A smoothing process applies to only the elements with high condition number of submatrices. The subject of this paper is to improve the convergence property of the ICCG method, but not to improve the shape of all elements in a whole analysis region by a smoothing method.

\section{Smoothing BASEd On Submatrix CONDITION Number}

\section{A. New Mesh Smoothing Scheme}

It is unnecessary to apply a mesh smoothing process to all elements in a whole analysis region, and the mesh smoothing process should be effectively applied to ill-conditioned elements which deteriorate the convergence property of the ICCG method. In order to smooth such elements, not the shape of the individual elements but the condition number of the submatrices of the coefficient matrix derived with the edge-based FEM has to be evaluated. Indeed, most of common smoothing methods move nodes to improve the shape of all the elements. In the proposed mesh smoothing scheme, nodes to be moved are chosen based on the condition number of the submatrix of the coefficient matrix. The procedure of the proposed smoothing scheme is described below.

Step 1) Create an initial mesh (e.g. using commercial software).

Step 2) Make a whole coefficient matrix derived from the initial mesh with the edge-based FEM.

Step 3) Subdivide the whole coefficient matrix into submatrices whose dimension is $\sim 1,000$, as shown in Fig. 1 . When the dimension of the submatrices is 
greater than 1,000 , it takes a long time to calculate the condition number of the submatrices.

Step 4) Calculate the condition number $\kappa$ of all the submatrices:

$$
\kappa_{i}=\frac{\mu_{\max i}}{\mu_{\min i}}
$$

where $\mu_{\max i}$ and $\mu_{\min i}$ are the maximum and minimum eigenvalue of $i$ th submatrix, respectively [9].

Step 5) Choose edges corresponding to submatrices with higher condition number than a threshold $\kappa_{\mathrm{th}}$. Here, the threshold $\kappa_{\text {th }}$ has to be appropriately decided so as to apply a mesh smoothing process to only nodes to be smoothed.

Step 6) Apply the Laplacian smoothing to nodes connecting with the chosen edges.

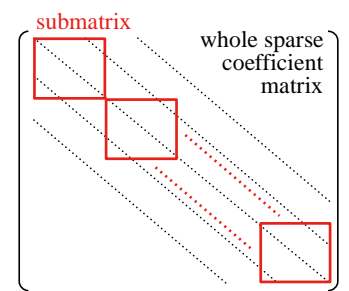

Fig. 1. Subdivision of whole sparse symmetric coefficient matrix. The whole coefficient matrix is subdivided into many submatrices whose dimension is $100 \sim 1,000$. A dot represents a nonzero entry in the matrix. The diagonal entries of a submatrix correspond to a part of the diagonal entries of the whole matrix and a nonzero entry is not overlapped on plural submatrices.

If a mesh smoothing method applies to all elements in a whole analysis region, elements of good quality are often deteriorated in exchange for improvement of elements of bad quality. Not all the elements of bad quality are necessarily the reason of bad convergence of the ICCG method.

Moreover, when a mesh smoothing process applies to a huge number of elements in the whole analysis region, a high computation cost is required. It is, therefore, important to appropriately decide the dimension of the submatrix. The larger the submatrix is, the longer time it takes to calculate its condition number. However, the dimension of the submatrix should not be too small to evaluate the property of the elements. $100 \sim 1,000$ in size of the submatrix is suitable from the viewpoint of computation time. The mesh smoothing method applies only to the elements corresponding to the submatrix of the higher condition number than a criteria. Meanwhile, it is unnecessary to smooth the other elements which correspond to submatrix of low condition number, because the convergence property of the ICCG method is not improved by smoothing them.

\section{B. Nodes to be smoothed}

In the proposed smoothing scheme, an important key is a way to choose nodes to be smoothed. Although tetrahedral mesh is employed in applications mentioned later in this paper, triangular mesh is used for a simple explanation of choosing the nodes to be smoothed.

The edge-based FEM yields a coefficient matrix with entries according to edge number. If there are triangular elements as shown in Fig. 2(a), the coefficient matrix of Fig. 2(b) is produced. The number of elements and edges are 13 and 24, respectively, so a 24 x 24 sparse coefficient matrix is derived with the edge-based FEM.

The coefficient matrix is subdivided into four $6 \times 6$ submatrices as shown in Fig. 2(b). Here, some entries of the whole matrix are not considered because they are out of the submatrices. The condition number of each submatrix is obtained from (1). Now, it was assumed that the condition number of the submatrix 3 was the highest. The submatrix 3 consists of the entries related to the edges $13 \sim 18$, as shown in Fig. 2(b). The related edges surely connect with two nodes, and these nodes are moved with a common smoothing method. In this simple example, six nodes red-highlighted in Fig. 2(a) are chosen. Of course, a chosen node on a boundary can be moved on the boundary. (a)

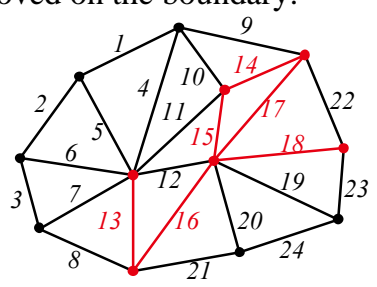

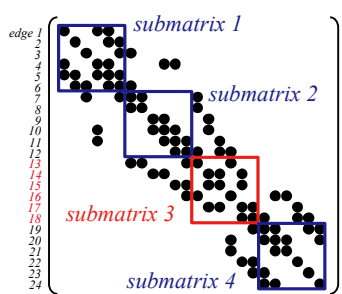

Fig. 2. (a) Simple triangular mesh. A red-highlighted number represents an edge related to the submatrix 3. A red-highlighted node represents a node connected by the red-highlighted edge. (b) 24 x 24 sparse coefficient matrix derived from simple triangular mesh with edge-based FEM. A dot represents a nonzero entry of the matrix. The coefficient matrix is subdivided into four $6 \times 6$ submatrices to calculate the condition number. It was assumed that the submatrix 3 had the highest condition number.

\section{APPLICATIONS}

\section{A. Simple Test}

The proposed smoothing scheme is applied to a simple test model consisting of a cubic permanent magnet $(1.0 \mathrm{~T})$. Fig. 3(a) shows the simple test model, and an initial tetrahedral mesh of the simple test model is generated with commercial software. When to generate the initial mesh some bad-shaped elements (such as flat and needle-like elements) are purposely made in air region for investigation. Fig. 3(b) shows the initial mesh containing the purposely distorted elements. The number of the nodes, edges, and elements of the initial mesh are 95, 444, and 286, respectively.

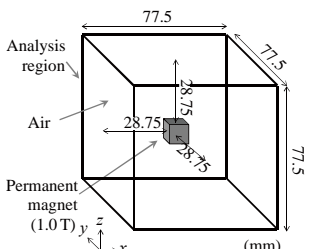

(a)

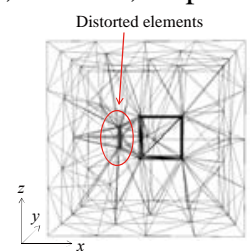

(b)

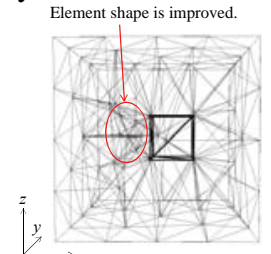

(c)
Fig. 3. (a) Simple test model consisting of one cubic permanent magnet (1.0 $\mathrm{T}$ ). The size of the analysis region is $77.5 \mathrm{~mm} \times 77.5 \mathrm{~mm} \times 77.5 \mathrm{~mm}$ and the permanent magnet $(20.0 \mathrm{~mm} \times 20.0 \mathrm{~mm} \times 20.0 \mathrm{~mm})$ is at the center of the analysis region. (b) Initial mesh of simple test model. Some distorted elements are purposely generated in the air region near the magnet. (c) Improved mesh of simple test model. The shape of flat elements are improved after applying the Laplacian smoothing process to the nodes corresponding to the submatrix 2 which has the worst condition number among all the submatrices. 
In order to apply the proposed smoothing scheme to the simple test model, 5 submatrices are produced by subdividing the whole coefficient matrix derived with the edge-based FEM. Fig. 4 shows the condition number of all the submatrices for the initial mesh. The submatrix 2 has the worst condition number among all the submatrices. Therefore, the nodes corresponding to the submatrix 2 are smoothed with the Laplacian smoothing method. Fig. 4 also shows the condition number of all submatrices after smoothing the chosen node. In spite of the smoothing of only the nodes corresponding to the submatrix 2, the condition number of the other submatrices is also improved, because a part of nodes corresponding to the submatrix 2 are shared with the other submatrices.

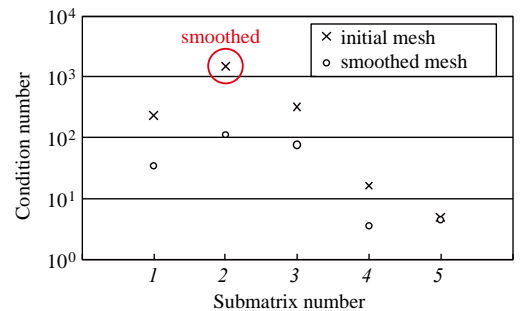

Fig. 4. Condition number of all submatrices both initial and smoothed mesh.

Fig. 3(c) shows the mesh of the simple test model after the proposed smoothing process. The flat elements of the initial mesh are improved. Table I shows the condition number of the whole coefficient matrix and the number of the ICCG iterations of both the initial and the smoothed mesh. It is known that the number of the ICCG iterations is reduced with decrease of the condition number of the whole coefficient matrix [9]. Both the condition number of the whole coefficient matrix and the number of the ICCG iterations after the smoothing process could be decreased as compared with those of the initial mesh.

TABLE I

CONDITION NUMBER OF WHOLE MATRIX AND NUMBER OF ICCG ITERATIONS

\begin{tabular}{lrr}
\hline & Condition number & Number of ICCG iterations \\
\hline Initial mesh & 2225.9 & 32 \\
Smoothed mesh & 225.1 & 21 \\
\hline \hline Reduction rate & $88.8 \%$ & $34.4 \%$ \\
\hline
\end{tabular}

\section{B. Laminated Iron Core Model}

The proposed smoothing scheme is applied to a more complicated model. Fig. 5 shows the laminated iron core model consisting of a rectangular laminated core and a coil, and an initial mesh generated with commercial software for electromagnetics. In order to analyze the magnetic influence of the iron lamination, it is desired to mesh the iron core with taking into account lamination structure [12]. Since a huge number of very thin iron plates are meshed, the initial mesh contains much many flat and needle-like elements in the laminated core and a portion of the coil, and many flat elements not depicted are also generated in the air region. The flat elements deteriorate the convergence property of an iterative solver. The number of the nodes, edges, and elements of the mesh are 268,368, 1,775,934, and 1,464,285, respectively.

The dimension of the whole coefficient matrix derived with the edge-based FEM is 1,775,934. The whole coefficient matrix is divided into 1,776 submatrices with dimension of approximately 1,000. Fig. 6 shows the condition number of all the submatrices for the initial mesh. The condition number of the submatrices are mostly distributed to high and low value. Fig. 7 shows an aspect ratio $\lambda$ for the condition number of each submatrix, where the aspect ratio $\lambda$ is the worst ratio between the radius of the inscribed and that of the circumscribed sphere of the elements corresponding to each submatrix;

$$
\lambda_{i}=\max _{j \in S_{i}} \frac{r_{\mathrm{c} i j}}{r_{\text {inj }}}
$$

where $r_{\mathrm{ci}}$ and $r_{\text {in }}$ are the radius of the circumscribed and the inscribed sphere, and $S_{i}$ is a set of elements corresponding to $i$ th submatrix, respectively. As shown in Fig. 7, the elements of the initial mesh are much ill-conditioned in whole. However, some submatrices with worse aspect ratio reveal a low condition number. Rather, some submatrices with better aspect ratio reveal a higher condition number. In terms of the edge-based FEM, it is hard to evaluate the shape quality of elements geometrically.

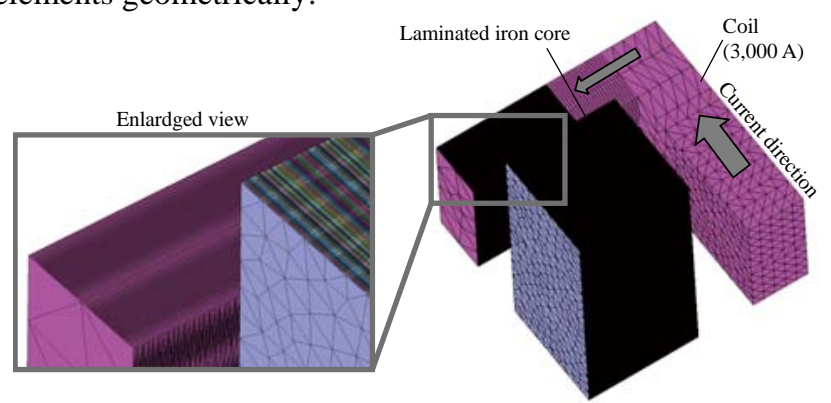

Fig. 5. Schematic view of laminated iron core model and initial tetrahedral mesh. 1/8 model is simulated because of symmetry. Elements in air are not drawn.

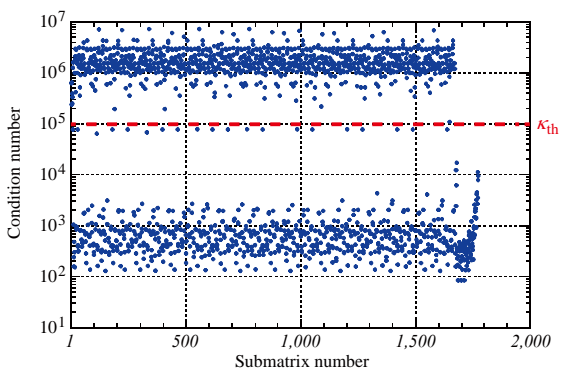

Fig. 6. Condition number of all submatrices in laminated iron core problem.

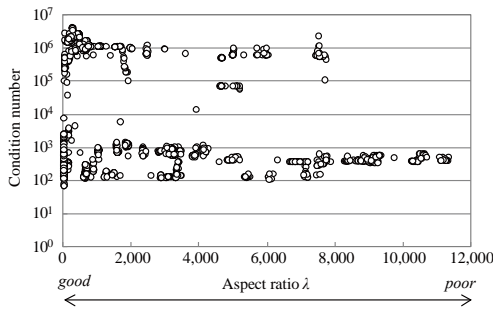

Fig. 7. Aspect ratio $\lambda$ for condition number of each submatrix. The best aspect ratio $\lambda$ is $1 / 3$.

In this problem, the threshold $\kappa_{\text {th }}$ is empirically decided to be $10^{5}$. Fig. 8 shows the elements corresponding to the submatrices whose condition number is higher than $\kappa_{\mathrm{th}}=10^{5}$. The shape of the elements shown in Fig. 8 is improved with 
the Laplacian smoothing method. Fig. 9 shows the higher condition number of the submatrices than the threshold $\kappa_{\mathrm{th}}=10^{5}$ for both the initial and the smoothed mesh. It was impossible to eliminate all the submatrices of higher condition number than the threshold $\kappa_{\mathrm{th}}=10^{5}$, however the condition number became small entirely. It was achieved that the submatrices with extremely high condition number $\left(>5 \times 10^{6}\right)$ disappeared by smoothing the elements corresponding to the submatrices of high condition number.

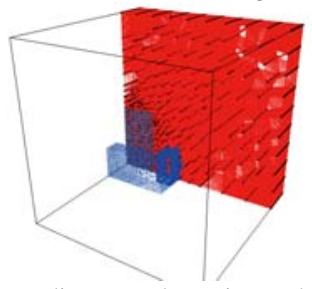

Fig. 8. Elements corresponding to submatrices whose condition number is higher than threshold $\kappa_{\text {th }}$. The chosen elements are concentrated on the coil, the laminated iron core, and the air around these objects.

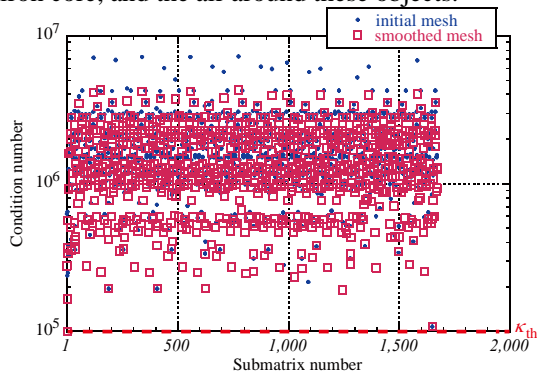

Fig. 9. Transition of condition numbers before and after the smoothing process. It was not achieved that all the condition numbers became less than the threshold $\kappa_{\mathrm{th}}=10^{5}$, however the extremely high condition numbers $\left(>5 \times 10^{6}\right)$ were eliminated.

In order to confirm the improvement of the convergence property of the ICCG method, the laminated iron core model was solved with the edge-based FEM. Table II shows the number of iterations for nonlinearity computation and total ICCG iterations. The number of total ICCG iterations after the smoothing process is 139,652 , whereas 155,361 iterations with the initial mesh. The proposed smoothing scheme reduced $10.1 \%$ of the number of total ICCG iterations and $9.2 \%$ of the computation time for the ICCG solver, without changing the dimension of the coefficient matrix. Here, it took approximately $1 \mathrm{~min}$. for the smoothing process including the calculation of the condition number of submatrices and the Laplacian smoothing. Therefore, the usefulness of the proposed smoothing scheme is also confirmed for the laminated iron core model. In this problem, it is impossible to calculate the condition number of the whole coefficient matrix because of a large size of its dimension.

\section{CONCLUSION}

In this paper, we have proposed a new smoothing scheme utilizing the condition number of submatrices into which a whole coefficient matrix derived with the edge-based FEM subdivides. In the proposed smoothing scheme, a common smoothing method applies to elements corresponding to submatrices of higher condition number than a threshold $\kappa_{\mathrm{th}}$.
As a result, by improving the condition number of the coefficient matrix derived with the edge-based FEM, the number of the ICCG iterations and the computation time can be reduced without changing the number of elements.

The proposed smoothing scheme was applied to a simple test model and a laminated iron core model. It was achieved that the number of the ICCG iterations and the computation time were reduced. For these models, the usefulness of the proposed scheme is confirmed. However, the condition number of the coefficient matrix depends on the FE formalism, the partial differential equation, the field singularity as well as the element shape. The proposed method is effective only in the only element shape. Therefore, when there is a room to improve the element shape, the propose method can shorten the computation time.

In future, it is necessary to develop both an element evaluation method in terms of geometrical shape and a smoothing method suitable for the edge-based FEM.

TABLE II

NONLINEARITY AND TOTAL ICCG ITERATIONS AND SOLVER TIME

\begin{tabular}{|c|c|c|c|}
\hline & $\begin{array}{r}\text { Nonlinearity } \\
\text { iterations }\end{array}$ & $\begin{array}{r}\text { Total ICCG } \\
\text { iterations }\end{array}$ & $\begin{array}{r}\text { ICCG solver } \\
\text { time }\end{array}$ \\
\hline Initial mesh & 10 & 155,361 & $11 \mathrm{~h} 37 \mathrm{~m}$ \\
\hline Smoothed mesh & 10 & 139,652 & $10 \mathrm{~h} 33 \mathrm{~m}$ \\
\hline Reduction rate & - & $10.1 \%$ & $9.2 \%$ \\
\hline
\end{tabular}

Processor: Intel Core 2 Duo E7500 2.93 GHz

\section{REFERENCES}

[1] C. R. S. Nunes, P. C. G. Mayrink, R. C. Mesquita, and D. A. Lowther, “A parallel remeshing method,” IEEE Trans. Magn., vol. 47, no. 5, pp. 1202-1205, May 2011.

[2] Y. Zhao, S. Niu, S. L. Ho, W. N. Fu, and J. Zhu, "A parameterized mesh generation and refinement method for finite element parameter sweeping analysis of electromagnetic devices," IEEE Trans. Magn., vol. 48, no. 2, pp. 239-242, Feb. 2012

[3] S. Matsutomo, S. Noguchi, and H. Yamashita, "Adaptive mesh generation method utilizing magnetic flux lines in two-dimensional finite element analysis,” IEEE Trans. Magn., vol. 48, no. 2, pp. 527-530, Feb. 2012.

[4] D. A. Field, "Laplacian smoothing and Delaunay triangulations," Communications in Numerical Methods in Engineering, vol. 4, pp. 709712, 1988

[5] T. Yokoyama, V. Cingoski, K. Kaneda, and H. Yamashita, “3-D Automatic Mesh Generation for FEA Using Dynamic Bubble System,” IEEE Trans. Magn., vol. 35, no. 3, pp. 1318-1321, May 1999.

[6] I. Tsukerman, "Approximation of conservative fields and the element ‘edge shape matrix’,” IEEE Trans. Magn., vol. 34, no. 5 pp. 3248-3251, Sep. 1998.

[7] I. Tsukerman and A. Plaks, "Comparison of accuracy criteria for approximation of conservative field on tetrahedral," IEEE Trans. Magn., vol. 34, no. 5, pp. 3252-3255, Sep. 1998.

[8] P. M. Knupp, “Algebraic mesh quality metrics," SIAM Journal on Scientific Computing, vol. 23, no. 1, pp. 193-218, 2001.

[9] A. Takada, S. Noguchi, and H. Igarashi, "Mesh quality investigation to improve convergence property of ICCG method for finite element method," Proc. 18th COMPUMAG, Jul. 2011.

[10] H. Igarashi, "On the property of the curl-curl matrix in finite element analysis with edge elements," IEEE Trans. Magn., vol. 37, no. 5, pp. 3129-3132, 2001.

[11] A. Takada, S. Noguchi, and H. Igarashi, "A new acceleration factor decision method for ICCG method based on condition number," IEEE Trans. Magn., vol. 48, no. 2, pp. 519-522, Feb. 2012.

[12] M. Miwa, T. Yamada, and S. Wakao, "Investigation of algebraic multigrid method for magnetic field analysis of an electric machine with a laminated core,” IEEE Trans. Magn., vol. 45, no. 3, pp. 992-995, Mar. 2009. 